\title{
Performance of the deuteron mass reconstruction with the AMS-02 RICH detector
}

\section{E. F. Bueno}

University of Groningen

E-mail: e.ferronato.buenodrug.nl

M. Vecchi*

University of Groningen and University of São Paulo

E-mail: m.vecchierug.nl

\begin{abstract}
The Alpha Magnetic Spectrometer (AMS-02) is operating aboard the International Space Station since May 2011, measuring cosmic rays in the GeV to TeV energy range. The isotopic composition of cosmic ray nuclei is strongly connected to their propagation in the Milky Way. Deuterons can be efficiently separated from the background of protons and helium nuclei by means of their mass, reconstructed by combining the velocity, charge and momentum, measured by different subdetectors, namely the RICH, the Time of Flight and the tracker. In this work, we will present our deuteron identification strategy, based on the mass reconstruction with the AMS-02 Ring Imaging CHerenkov ( $\mathrm{RICH}$ ). Our method aims at identifying with great precision the signal among the background of protons and heavier nuclei fragmenting inside the AMS-02 detector.
\end{abstract}

36th International Cosmic Ray Conference -ICRC2019-

July 24th - August 1st, 2019

Madison, WI, U.S.A.

\footnotetext{
* Speaker.
} 


\section{Introduction}

\subsection{Deuterons in cosmic rays}

The presence of deuterons in CRs is mainly due to the interactions of certain primary cosmic rays (CRs), namely $p,{ }^{3} \mathrm{He}$ and ${ }^{4} \mathrm{He}$, with the interstellar medium. Therefore, the measurement of the deuteron flux and secondary-to-primary ratios, such as the deuteron-to-proton $(D / p)$ flux ratio, provide valuable insight into the propagation processes of CRs in the galaxy [1].

In the past decades, many experiments have studied the deuteron flux and the $D / p$ ratio, but above $1 \mathrm{GeV} / \mathrm{n}$ the measurements are scarce and affected by large uncertainties. The Alpha Magnetic Spectrometer (AMS-02) has the capability to perform these measurements with great precision in this range by using its Time of Flight and Cherenkov detector. In this work we present the method for identifying deuterons in CRs and assess the performance of the detector in terms of its mass resolution as a function of the kinetic energy per nucleon using data collected by the AMS-02 during 4 years, from May 2011 to May 2015.

\subsection{The Alpha Magnetic Spectrometer}

AMS-02 is a general-purpose, state-of-the-art magnetic spectrometer installed and continuously taking data aboard the International Space Station (ISS) since May 2011 [2]. It was designed to identify and measure the properties of charged particles in the $\mathrm{GeV}$ to $\mathrm{TeV}$ energy range.

AMS-02 is composed of several sub-detectors: the nine layers of the silicon tracker [3], together with the $0.14 \mathrm{~T}$ permanent magnet, measure the charge, charge sign and the rigidity ( $R=p c / Z e$ ) of the particle; the Transition Radiator Detector (TRD) [4] is used to separate leptons from hadrons; four Time of Flight (TOF) [5] scintillator planes are used to measure the charge and velocity of the particle and act as the main trigger of the experiment; the Anti-Coincidence Counter (ACC) [6] is used to reject particles with high incidence angle; the Ring Imaging Cherenkov Detector (RICH) also measures the velocity and charge [7]; finally, the Electromagnetic Calorimeter (ECAL) [8] is used for lepton-hadron separation and the measurement of the energy of the particle.

\subsection{The RICH detector}

The RICH detector [9] has three main components: a radiator plane, in which the incoming charged particles produce Cherenkov radiation when traversing it; a surrounding mirror, with the purpose of reflecting the Cherenkov photons of particles with high incidence angle; and the detection plane, a matrix of photomultiplier tubes (PMTs) where the Cherenkov photons are detected.

The detection matrix is composed of $6804 \times 4$ Hamamatsu R7600-00-M16 multi-anode PMTs, with a final effective spatial granularity of $8.5 \times 8.5 \mathrm{~mm}^{2}$. One important feature of this plane is the presence of a hole in its center,in order to minimize the interaction of the particles before they reach the ECAL, with an area of $64 \times 64 \mathrm{~cm}^{2}$. The radiator plane is made of 16 squared sodium fluoride (NaF) tiles with sides of $11.4 \mathrm{~cm}$ and $5 \mathrm{~mm}$ thick. The NaF is surrounded by 92 square aerogel tiles, with sides of the same size side and with thickness of $2.5 \mathrm{~cm}$. The aerogel has a refractive index $n=1.05$, while the sodium fluoride has a larger refractive index $n=1.34$, hence the Cherenkov cones produced in the center of the radiator plane are broader and avoid the hole in the detection plane. The different refractive indices also make the two materials suitable for measuring complementary velocity ranges: the threshold for Cherenkov light production for the $\mathrm{NaF}$ 
is $\beta_{T h}=0.78$ and for the aerogel $\beta_{T h}=0.96$. In this work, the NaF is used to measure the velocity of particles in the range $0.75<\beta<0.97$ and the aerogel is used in the range $0.96<\beta<0.996$, while the corresponding range in kinetic energy per nucleon goes from 0.5 to $10 \mathrm{GeV} / \mathrm{n}$.

\section{Deuteron identification}

\subsection{Event selection}

A quality selection is applied to the data to ensure any that background other than protons is removed. Only downgoing particles within the acceptance of the TOF, the first eight layers of the tracker and the RICH are allowed. The TOF allows for the selection of downgoing particles and the reconstructed tracker track enables the rejection of particles which are not in the acceptance of the mentioned subdetectors.

In order to select single-charged particles, several independent measurements made along the trajectory of the particle are taken into account. The TRD, the first layer of the tracker, the inner tracker (layers 2-8), and the four layers of the TOF are used for that purpose. The usage of multiple independent charge measurements is of great importance for this analysis. Heavier nuclei, mainly helium (given its abundance in CRs), can fragment inside the detector and leave spurious signals of $Z=1$. By measuring the charge from the first layer of the tracker until the last layer of the TOF, located just above the RICH, we ensure that the background due to heavier nuclei is greatly reduced, and can be considered as negligible.

On top of that, only galactic CRs are selected. For a given measurement of $\beta$, the mass and thus the momentum is different for protons a deuterons: due to their lower mass, the rigidity of protons is lower. For every event we calculate the proton rigidity using the measured velocity under assumption that the particle mass is equal to the proton mass. Only events with the proton rigidity above the geomagnetic cutoff within the AMS-02 field of view are selected.

\subsection{Mass measurement}

In AMS-02 the mass is identified by combining the momentum and velocity measurements:

$$
m=\frac{R Z}{\beta \gamma}
$$

Where $\gamma$ represents the Lorentz factor; $R$ is the rigidity; $\beta$ the velocity divided by the speed of light; and $Z$ is the charge. The uncertainty relative to $m$ is given by the expression:

$$
\left(\frac{\Delta m}{m}\right)^{2}=\left(\frac{\Delta R}{R}\right)^{2}+\gamma^{4}\left(\frac{\Delta \beta}{\beta}\right)^{2}
$$

Due to the presence of the $\gamma^{4}$ factor in this equation, the mass resolution has two distinct regimes: at low rigidities, $\beta$ is lower and therefore $\gamma^{4}$ becomes negligible, hence $\Delta m / m$ is dominated by the rigidity resolution. Conversely, at high rigidities, $\beta \approx 1$, hence $\gamma^{4}$ is larger and $\Delta m / m$ is dominated by the velocity resolution. In this work, we exploit these features to study $\Delta \beta / \beta$, $\Delta R / \Delta R$ and $\Delta m / \Delta m$. 


\section{Mass reconstruction performance}

\subsection{Velocity resolution}

The velocity resolution must be studied using a sample of events whose rigidity measurement is well under control, for which the mass resolution (equation 2.2) is dominated by $\gamma^{4}$. Hence, only events with rigidity $R$ in the interval between 50 and $100 \mathrm{GV}$ were considered.

The estimation of the resolution was done through a double-gaussian fit performed around the center of the distribution, in a range containing $90 \%$ of the events. The resolution is then given by the second moment of this sum of gaussian functions. This process was done for both RICH radiators. Figure 1 shows an example of such fit in the NaF region. In order to assess the errors related to the calculation of $\Delta \beta / \beta, 1000$ toy Monte Carlo histograms were generated based on the original distribution and the fitting procedure was repeated in each of them. The final value of $\Delta \beta / \beta$ was then the average of these results.

$$
\beta: 50<\mathrm{R}<100 \mathrm{GV}(\mathrm{NaF})
$$

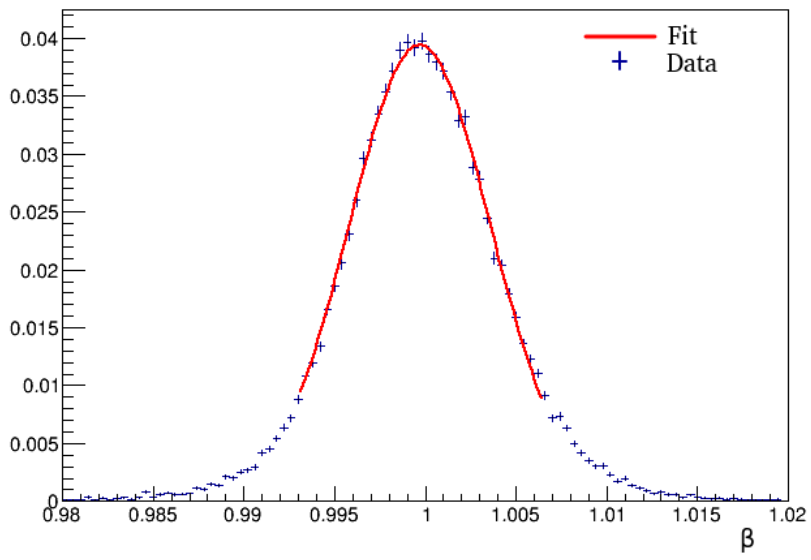

Figure 1: NaF velocity distribution for simulated protons events with rigidity between 50 and $100 \mathrm{GV}$. The red line shows the results of a fit to a double Gaussian.

This process was done for both simulated events and data. Results are shown in table 1. Both radiators have sufficient accuracy to measure the velocity of the particles with uncertainties below the percent level. The NaF has an slightly inferior resolution due to the fact that the Cherenkov cones produced in this radiator larger. In both cases the simulations are in good agreement with data.

\begin{tabular}{lcc}
\cline { 2 - 3 } & \multicolumn{2}{c}{$\mathrm{NaF}$} \\
\cline { 2 - 2 } & \multicolumn{2}{c}{$\Delta \beta / \beta$} \\
\hline Data & $(3.6 \pm 0.1) \times 10^{-3}$ & $(1.2 \pm 0.1) \times 10^{-3}$ \\
\hline MC & $(3.5 \pm 0.1) \times 10^{-3}$ & $(1.2 \pm 0.2) \times 10^{-3}$ \\
\hline
\end{tabular}

Table 1: $\Delta \beta / \beta$ values for the $\mathrm{NaF}$ and aerogel radiators for both data and simulated proton events. 


\subsection{Rigidity resolution}

The rigidity resolution was studied using a sample of events with $1.05<R<22 \mathrm{GV}$, divided in bins of kinetic energy per nucleon.

Similarly to what was done for the velocity, the rigidity resolution is determined by using a double-gaussian fit on toy MC histograms. However, to estimate the rigidity resolution, the distribution of the inverse rigidity is studied, because this is the quantity actually measured, thus is the quantity expected to have a gaussian behavior [11]. Since the analysis is done in velocity bins, deuterons and protons will have different values of $R$, therefore the distributions have two peaks, one for each isotope. The fits are then performed around the peak of protons, in a range containing $90 \%$ of the events. An example of fit is shown in figure 2.

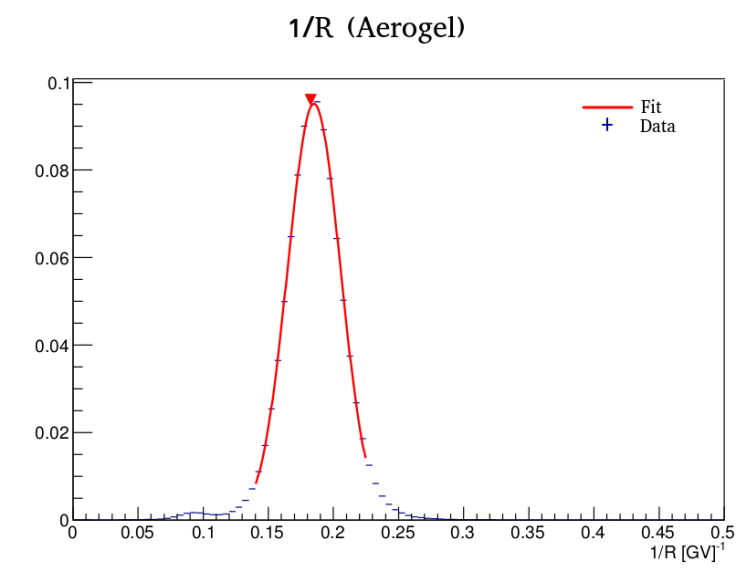

Figure 2: Aerogel $1 / R$ distribution for experimental data with kinetic energy per nucleon between 4.587 and 5.055. The red line shows the results of a fit to a double Gaussian.

In order to compare data and MC properly, the MC samples are a mixture of $98 \%$ proton and $2 \%$ deuteron simulated events. These weights are chosen to reproduce the known isotopic composition of protons in CRs. Results are shown in figure 3. As expected, at the lowest rigidities the resolution is worse, with values of $\Delta R / R$ as high as $10 \%$, due to the fact that processes such as multiple Coulomb scattering become more relevant. In both cases MC and data are in good agreement.

\subsection{Mass resolution}

The process for calculating the mass resolution is essentially the same as the one done for the rigidity. However, the fit is performed on $1 / m$ distributions. The choice for $1 / m$ comes from equation 2.1: since $1 / R$ is Gaussian and the RICH measures $\beta, 1 / m$ is Gaussian-like at first approximation. Results are shown in figure 4 . The mass resolution varies from $7 \%$ to $17 \%$ in the studied range, with good agreement between data and simulations. Comparing to figure 2, it is possible to note that the mass resolution worsens in a region where the rigidity resolution has a flat behavior: as already discussed, at higher energies $\Delta \beta / \beta$ becomes more relevant and it is what ultimately limits the range where these measurements can be made. 


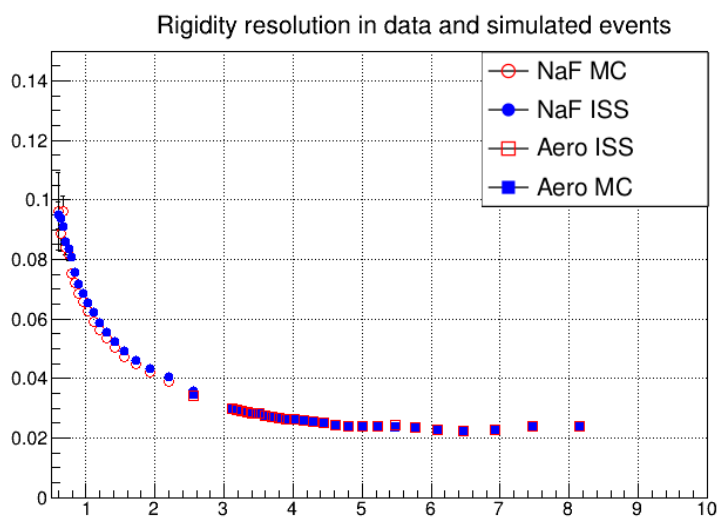

Figure 3: Rigidity resolution as a function of the kinetic energy per nucleon for data (filled blue) and MC (empty red), for both $\mathrm{NaF}$ (circles) and aerogel (squares).

A similar study has been made for the mass identification of helium isotopes with AMS-02 [10], showing a similar behavior. However, the performance for helium isotopes is globally better, due to the fact that the RICH has a better velocity resolution for particles with $Z \geq 2$ [9].

\section{$\Delta \mathrm{m} / \mathrm{m}$ - Data and $\mathrm{MC}$}

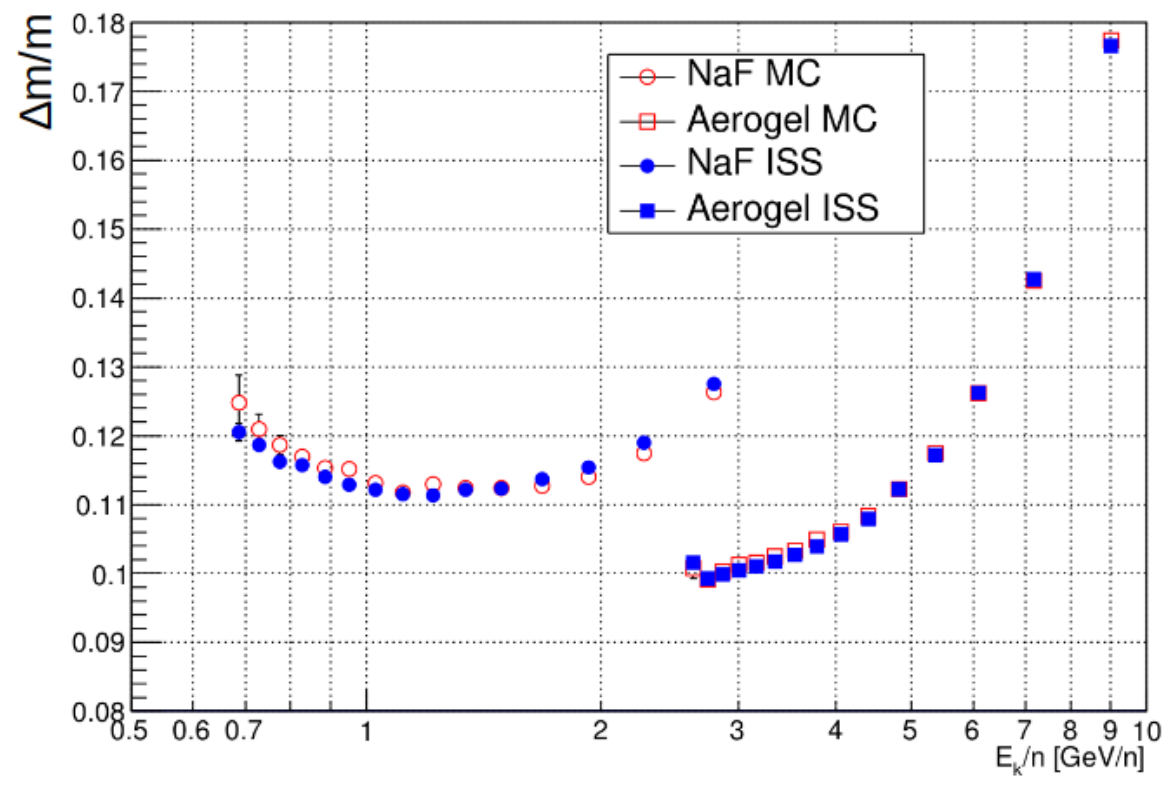

Figure 4: Mass resolution as a function of the kinetic energy per nucleon for data (filled blue) and MC (empty red), for both $\mathrm{NaF}$ (circles) and aerogel (squares).

\section{Conclusion}

An evaluation of the performance of AMS-02 RICH for the detection of deuterons has been presented: the mass, rigidity and velocity resolutions have been studied in both data and simulated 
events. The dependence of the mass resolution on the momentum and velocity in different regimes was used to study their resolutions independently. Results showed that data and simulations are in agreement in all cases and that $\Delta m / m$ is up to $17 \%$ around $10 \mathrm{GeV} / \mathrm{n}$, allowing for a good separation of the isotopes. Beyond this energy, precise isotope separation is challenged by the velocity resolution.

\section{References}

[1] Coste et al., Astronomy \& Astrophysics, 539 (2012), A88.

[2] Kounine et al., International Journal of Modern Physics E, 218 (2012), 1230005.

[3] B. Alpat et al., Nucl. Instrum. Meth. A 613, 207 (2009)

[4] T. Kirn et al, Nucl. Instrum. Meth. A 706 (2013) 43-47

[5] V. Bindi et al, Nucl. Instr. Meth. A 743 (2014) 22-29

[6] Ph. von Doetinchem et al, Nucl. Phys. Proc. Suppl. 197 (2009) 15-18

[7] M. Aguilar-Benitez et al, Nucl. Instr. Meth. A 614 (2010) 237-249

[8] C. Adloff et al, Nucl. Instr. Meth. A 714 (2013) 147-154

[9] Liu et al., Nucl. Instr. Meth. Phys. Res. A, A876 (2017), 5-8.

[10] X. Xia and A. Oliva, Nucl. Instr. Meth. A 868 (2017) 139.

[11] G. Ambrosi et al., Nucl. Instr. Meth. A 869 (2017) 29. 